\title{
ANOTHER NOTE ON LEVINE'S DECOMPOSITION OF CONTINUITY
}

\author{
DAVID A. ROSE \\ Department of Mathematics \\ Oral Roberts University, Tulsa, OK 74171 \\ ROY A. MIMNA \\ 520 Meadowland Drive \\ Hubbard, $\mathrm{OH} 44425$ \\ DRAGAN JANKOVIĆ \\ Department of Mathematics \\ East Central University, Ada, OK 74820
}

(Received November 22, 1994 and in revised form January 30, 1995)

\begin{abstract}
Several decompositions of continuity each stronger than Norman Levine's are found improving results of $\mathbf{J}$. Chew and $\mathbf{J}$. Tong, as well as of the first two named authors above.

KEY WORDS AND PHRASES. Weak continuity, local interiority, local relative continuity, weak $\alpha$ continuity, conditions $(*)$ and $(* *)$.
\end{abstract}

1991 AMS SUBJECT CLASSIFICATION CODE. 54C10.

\section{INTRODUCTION.}

In [1] N. Levine introduced the notions of $w^{*}$ continuity and weak continuity, and showed that for an arbitrary function $f: X \rightarrow Y$ between topological spaces, $f$ is continuous if and only if it is both weakly continuous and $w^{*}$ continuous. In [2] $w^{*}$ continuity was replaced in this decomposition by the strictly weaker condition local $w^{*}$ continuity. Later, in [3] the decomposition was strengthened further by replacing weak continuity with weak $\alpha$-continuity, a condition strictly weaker than weak continuity introduced by $\mathrm{T}$. Noiri [4]. More recently, in [5] local $w^{*}$ continuity was replaced by the strictly weaker local relative continuity which in this paper is called basic relative continuity. It is interesting that relative continuity as introduced by Chew and Tong in [6] is equivalent to Levine's $w^{*}$ continuity. Its real merit however, lies in the fact that its basic (or local) version can replace local $w^{*}$ continuity in many applications while being strictly weaker. In Section 3 of this paper, two new function conditions (*) and $(* *)$ are introduced each strictly weaker than basic relative continuity and with $(* *)$ strictly weaker than (*). It is shown that for any function $f: X \rightarrow Y$, the following are equivalent.

(a) $f$ is continuous.

(b) $f$ is weakly continuous and satisfies (**).

(c) $f$ is weakly $\alpha$-continuous and satisfies (*).

Of course, decomposition (c) improves the aforementioned result of [5]. Most notable though is the fact that every function into a regular space satisfies (*). Results of Levine [1] and Noiri [4] follow immediately. That is, weakly $(\alpha-)$ continuous functions into regular spaces are continuous. Decomposition (b) improves a result of Chew and Tong [6] that every weakly continuous function having interiority is continuous. In Section 2 we discuss the interiority condition and a basic version. First, recall the following definitions.

DEFINITION 1. A function $f: X \rightarrow Y$ is weakly continuous if $f^{-1}(V) \subseteq \operatorname{Int}\left(f^{-1}(C l(V))\right)$ for each (basic) open subset $V \subseteq Y$.

DEFINITION 2. A function $f: X \rightarrow Y$ is weakly $\alpha$-continuous if $f^{\alpha}: X^{\alpha} \rightarrow Y$ is weakly continuous where $X^{\alpha}$, the $\alpha$-space for $X$, has the same underlying set as $X$, and its topology is the 
smallest expansion of the topology on $X$ for which nowhere dense subsets of $X$ are closed, and where for each $x \in X, f(x)=f^{\alpha}(x)$.

DEFINITION 3. A function $f: X \rightarrow Y$ is basically relatively contimuous if there exists an open basis $B$ for the topology on $Y$ such that $f^{-1}(V)$ is open in the subspace $f^{-1}(C l(V))$ for each $V \in B$.

Weak $\alpha$-continuity was introduced by Noiri [4] and studied further in [3], and basic relative continuity was introduced in [5] under the name local relative continuity

\section{INTERIORITY OF CHEW AND TONG}

Throughout this paper, a function on a topological space $X$ into a topological space $Y$, will be represented by $f: X \rightarrow Y$, where $X$ and $Y$ have no particular properties unless otherwise indicated

DEFINITION 4. [6] A function $f: X \rightarrow Y$ has interiority if $\operatorname{Int}\left(f^{-1}(C l(V))\right) \subseteq f^{-1}(V)$ for each open set $V \subseteq Y$.

In [6] it is shown that weak continuity together with interiority implies continuity. The reader will immediately see that the condition of interiority is equivalent to the condition that for each open set $V \subseteq Y, f^{-1}(F r(V)) \cap I n t\left(f^{-1}(C l(V))\right)=\emptyset$. The following results illustrate some of the limitations of the interiority condition

THEOREM 1. Let $Y$ be any $T_{1}$ space. Then for any topological space $X$, and for any surjection $f: X \rightarrow Y$, $f$ has the interiority condition if and only if $Y$ is discrete.

PROOF. If $Y$ is discrete, then every function $f: X \rightarrow Y$ has interiority since for all $V \subseteq Y$, $C l(V)=V$ so that $\operatorname{Int}\left(f^{-1}(C l(V))\right)=\operatorname{Int}\left(f^{-1}(V)\right) \subseteq f^{-1}(V)$. Now suppose that $f: X \rightarrow Y$ is a surjection having interiority and suppose that points are closed in $Y$; that is, $Y$ is a $T_{1}$ space. If $y$ is a nonisolated point in $Y$, then $\{y\}$ is not open so that every open set containing $y$ contains points of $Y-\{y\}$. Then $V=Y-\{y\}$ is a dense open subset of $Y$. By interiority of the surjection $f$, $\operatorname{Int}\left(f^{-1}(C l(V))\right)=\operatorname{Int}\left(f^{-1}(Y)\right)=\operatorname{Int}(X)=X \subseteq f^{-1}(V)$. Thus, $f(X) \subseteq V$ and $f$ is not surjective. Hence each $y \in Y$ is isolated and $Y$ has the discrete topology.

The above argument can be adapted to yield the following results

COROLLARY 1. No function with a nondiscrete $T_{1}$ range subspace has interiority.

COROLLARY 2. The $T_{1}$ range of interiority function is a discrete subspace of the codomain. In particular, if $Y$ is a $T_{1}$ space and $f: X \rightarrow Y$ has interiority, then $f(X)$ is a discrete subspace of $Y$.

THEOREM 2. Let $X$ be nonempty and connected and let $Y$ be a $T_{1}$ space. Then every weakly contimuous function $f: X \rightarrow Y$ having interiority is constant.

PROOF. From [6], $f$ is continuous so that $f(X)$ is connected and discrete. Then each $\{y\}$ $\subseteq f(X)$ is clopen (closed and open) in the subspace $f(X)$, so that $f(X)$ is a nonempty singleton set.

A less rigid condition than interiority for a function is basic interiority.

DEFINITION 5. A function $f: X \rightarrow Y$ has basic interiority if there is an open base $\mathbf{B}$ for the topology on $Y$ such that $\operatorname{Int}\left(f^{-1}(C l(V))\right) \subseteq f^{-1}(V)$ for each $V \in \mathbf{B}$.

REMARK 1. It is easy to see that for any function, weak continuity plus basic interiority implies continuity so that the result of Chew and Tong [6] is improved. A further strengthening of this result is obtained in the next section where it is shown that basic interiority can be replaced by condition (**) which is not only weaker than basic interiority but is also weaker than continuity so that a new decomposition of continuity is obtained.

REMARK 2. If $Y$ is a space such that every open set is closed, then every function $f: X \rightarrow Y$ has interiority. If $Y$ is a zero-dimensional space (having a basis of clopen sets), then every function $f: X \rightarrow Y$ has basic interiority. It will be shown by example in the next section that basic interiority is strictly weaker than interiority.

\section{IMPROVED LEVINE DECOMPOSITIONS OF CONTINUITY}

Our first goal in this section is to obtain a decomposition of continuity into weak continuity plus a complementary condition weaker than basic relative continuity. If it happens that the complementary 
condition is satisfied by all functions whose codomain is regular, then a consequence will be the result of Norman Levine [1] that every weakly continuous function into a regular space is continuous.

DEFINITION 6. A function $f: X \rightarrow Y$ satisfies condition (*) if for each $x \in X$ and for each open set $V$ containing $f(x)$, there exists an open subset $V_{x} \subseteq V$ with $f(x) \in V_{x}$ such that $x \notin C L\left(f^{-1}\left(C L\left(V_{x}\right)\right)-f^{-1}(V)\right)$.

DEFINITION 7. A function $f: X \rightarrow Y$ satisfies condition (**) if for each $x \in X$ and for each open set $V$ containing $f(x)$, there exists an open subset $V_{x} \subseteq V$ with $f(x) \in V_{x}$ such that $x \notin C l\left(\operatorname{Int}\left(f^{-1}\left(C l\left(V_{x}\right)\right)\right)-f^{-1}(V)\right)$.

THEOREM 3. For any function $f: X \rightarrow Y$, basic relative continuity $\Rightarrow(*) \Rightarrow(* *)$ and basic interiority $\Rightarrow(* *)$

PROOF. It is clear that $(*) \Rightarrow(* *)$. To see that basic relative continuity implies $(*)$, let $\mathbf{B}$ be a basis for the topology on $Y$ such that for each $V \in \mathbf{B}, f^{-1}(V)$ is relatively open in $f^{-1}(C l(V))$. Now let $x \in X$ and let $V \subseteq Y$ be an open set containing $f(x)$. Choose $V_{x} \in \mathbf{B}$ with $f(x) \in V_{x}$ and $V_{x} \subseteq V$. By local relative continuity, there is an open set $U$ such that $f^{-1}\left(V_{x}\right)=U \cap f^{-1}\left(C l\left(V_{x}\right)\right)$. Thus, $U \cap f^{-1}\left(C l\left(V_{x}\right)-V_{x}\right)=\emptyset$, so that $U \cap f^{-1}\left(C l\left(V_{x}\right)-V\right)=\emptyset$. Since $x \in U$, $x \notin C l\left(f^{-1}\left(C l\left(V_{x}\right)\right)-f^{-1}(V)\right)$, and $f: X \rightarrow Y$ has $(*)$.

Now let $f: X \rightarrow Y$ be any function with basic interiority. Let $\mathbf{B}$ be an open basis for the topology on $Y$ such that for each $V \in \mathbf{B}, \operatorname{Int}\left(f^{-1}(C l(V))\right) \subseteq f^{-1}(V)$. If $x \in X$ and $V$ is an open set containing $f(x)$, choose $V_{x} \in \mathbf{B}$ with $f(x) \in V_{x}$ and $V_{x} \subseteq V$. By basic interiority, $\operatorname{Int}\left(f^{-1}\left(C l\left(V_{x}\right)\right)\right) \subseteq f^{-1}\left(V_{x}\right) \subseteq f^{-1}(V)$ so that $\operatorname{Int}\left(f^{-1}\left(C l\left(V_{x}\right)\right)\right)-f^{-1}(V)=\emptyset$. Evidently, $f$ satisfies condition $(* *)$ since $x \notin C l\left(\operatorname{Int}\left(f^{-1}\left(C l\left(V_{x}\right)\right)\right)-f^{-1}(V)\right)$.

The examples below show that none of the implications of the preceding theorem are reversible. But first we observe perhaps the most notable consequence of $(*)$

THEOREM 4. If $Y$ is a regular space, then every function $f: X \rightarrow Y$ satisfies condition (*).

PROOF. If $x \in X$ and $V$ is an open set containing $f(x)$, choose an open set $V_{x}$ containing $x$ such that $C l\left(V_{x}\right) \subseteq V$. Then $x \notin \emptyset=C l\left(f^{-1}\left(C l\left(V_{x}\right)\right)-f^{-1}(V)\right)$.

EXAMPLE 1. Let $R$ be the usual space of real numbers and $f: R \rightarrow R$ be the topologist's sine function defined by $f(x)=\left\{\begin{array}{cc}\sin \left(\frac{1}{x}\right), & \text { if } x \neq 0 \\ 0, & \text { if } x=0\end{array}\right]$. Since $R$ is regular, $f$ satisfies (*). However, $f$ is not basically relatively continuous. For if $V \subseteq\left(-\frac{1}{2}, \frac{1}{2}\right)$ is any basic open set containing 0 and if $a$ is the least upper bound of $V$, then $a \in C l(V)-V$, and $0 \in C l\left(f^{-1}(a)\right)$. Thus if $U$ is any open set in the domain containing $0, a \in f(U)$ so that $U \cap f^{-1}(C l(V)-V) \neq \emptyset . \quad$ Evidently, $U \cap f^{-1}(C l(V)) \neq f^{-1}(V)$ showing that $f^{-1}(V)$ is not relatively open in $f^{-1}(C l(V))$.

REMARK 3. An alternative to the function of Example 1 would be any non-continuous separately continuous function $f: R \times R \rightarrow R$ where $R$ is the usual space of real numbers. Since $R$ is regular $f$ has (*) but is not basically relatively continuous since it is a consequence of Corollary 3 of [5] that every separately continuous function $f: R \times R \rightarrow R$ is continuous if it is basically relatively continuous

EXAMPLE 2. We will see that (**) does not imply (*) by showing that basic interiority does not imply (*). Let $f: X \rightarrow Y$ be the identity function from $X$, the set of real numbers with the indiscrete topology onto $Y$, the set of real numbers endowed with the simple expansion of the usual topology by the set $Q$ of rational numbers. Since every basis for the topology on $Y$ must contain bounded open sets let us consider in particular a non-dense basic open set $V \subseteq Q$. Then $\operatorname{Int}\left(f^{-1}(C l(V))\right)=\emptyset \subseteq f^{-1}(V)$ showing that $f$ has basic interiority. On the other hand, $f$ does not satisfy condition (*) when the domain set is endowed with any subtopology of the usual topology. For if $U$ is any usual open set containing 0 , and if $V=(-1,1) \cap Q$, then $0=f(0) \in V$, yet for any open set $V_{0} \subseteq V$ with $0 \in V_{0}$, $U \cap f^{-1}\left(C l\left(V_{0}\right)-V_{0}\right) \neq \emptyset$. 
REMARK 4. The function $f$ of Example 2 does not have interiority since $Q$ is a proper open and dense set. Therefore, $\operatorname{Int}\left(f^{-1}(C l(Q))\right)=X$ which is not contained in $Q=f^{-1}(Q)$. Thus, the example also serves to prove that basic interiority is strictly weaker than interiority.

EXAMPLE 3. Let $f: X \rightarrow Y$ be the identity function from the space $X$ of real mumbers with the discrete topology onto the usual space of real numbers $Y$. Since $Y$ is regular, $f$ has (**). However, if $V$ is any bounded basic open subset of $Y, \operatorname{Fr}(V) \neq \emptyset$ so that $\operatorname{Int}\left(f^{-1}(C l(V))\right)=f^{-1}(C l(V))$ is not a subset of $f^{-1}(V)$.

The following decomposition of continuity improves the result of Chew and Tong [6] as well as Theorem 5 of [5].

THEOREM 5. A function $f: X \rightarrow Y$ is continuous if and only if $f$ is both weakly continuous and satisfies condition $(* *)$.

PROOF. The necessity is clear since continuity implies both weak continuity and basic relative continuity and basic relative continuity implies condition (**). For the sufficiency, let $x \in X$ and let $V$ be an open set containing $f(x)$. By (**), there is an open subset $V_{x} \subseteq V$ with $f(x) \in V_{x}$ such that $x \notin C l\left(\operatorname{Int}\left(f^{-1}\left(C l\left(V_{x}\right)\right)\right)-f^{-1}(V)\right)$. Thus, there exists an open set $U$ containing $x$ such that $U \cap \operatorname{Int}\left(f^{-1}\left(C l\left(V_{x}\right)\right)\right) \subseteq f^{-1}(V)$. By weak continuity, $f^{-1}\left(V_{x}\right) \subseteq \operatorname{Int}\left(f^{-1}\left(C l\left(V_{x}\right)\right)\right)$ and since $x \in f^{-1}\left(V_{x}\right), W=U \cap \operatorname{Int}\left(f^{-1}\left(C l\left(V_{x}\right)\right)\right)$ is an open set containing $x$ and $f(W) \subseteq V$. This shows that $f$ is continuous at each $x \in X$.

COROLLARY 3. [1] Every weakly contimuous function into a regular space is continuous.

REMARK 5. Actually, a space $Y$ is regular if and only if every weakly continuous function $f: X \rightarrow Y$ is continuous. To see this let $y \in Y$ be a non-regular point, i.e., $y$ has an open neighborhood $V$ such that for every open set $W$ containing $y, C l(W)-V \neq \emptyset$. If $\sigma$ is the topology on $Y$, let $\sigma^{*}$ be an associated topology having for a subbase $\left\{C l_{\sigma}(W) \mid y \in W \in \sigma\right\} \cup\{W-\{y\} \mid W \in \sigma\}$, and let $f:\left(Y, \sigma^{*}\right) \rightarrow(Y, \sigma)$ be the identity function. Then $f$ is weakly continuous being continuous at each $z \in Y-\{y\}$ and weakly continuous at $y$. But, $f$ is not continuous at $y$ since $y \notin \operatorname{Int}_{\sigma^{*}} f^{-1}(V)$. In particular, this observation makes clear the futility of looking for a continuity-complement to weak continuity weaker than $(* *)$ which would hold for all functions with codomain in a class larger than the class of regular spaces.

The above decomposition of continuity can be modified as follows.

THEOREM 6. A function $f: X \rightarrow Y$ is contimuous if and only if $f$ is weakly $\alpha$-continuous and satisfies condition (*).

PROOF. The necessity is clear. For the sufficiency, let $f^{\alpha}: X^{\alpha} \rightarrow Y$ be the function defined by $f^{\alpha}(x)=f(x)$ for each $x \in X$, and note that $f^{\alpha}$ has (*) whenever $f$ has (*). If also $f$ is weakly $\alpha$ continuous, then $f^{\alpha}: X^{\alpha} \rightarrow Y$ is continuous so that by a result of Noiri [4], $f: X \rightarrow Y$ is weakly continuous. Then by the above decomposition of continuity, $f$ is continuous.

Theorem 6 strengthens Theorem 6 of [5] and yields the following result of Noiri [4].

THEOREM 7. Every weakly a-continuous function into a regular space is continuous.

\section{REFERENCES}

[1] LEVINE, N., A decomposition of continuity in topological spaces, Amer. Math. Monthly 68 (1961), 44-46.

[2] ROSE, D., On Levine's decomposition of continuity, Canad. Math. Bull. 21 (1978), 477-481.

[3] ROSE, D., A note on Levine's decomposition of continuity, Indian J. Pure Appl. Math. 21 (1990), 985-987.

[4] NOIRI, T., Weakly $\alpha$-continuous functions, Int. J. Math. \& Math. Sci. 10 (1987), 483-490.

[5] MIMNA, R. and ROSE, D., On local relative continuity, to appear in the Real Analysis Exchange.

[6] CHEW, J. and TONG, J., Some remarks on weak continuity, Amer. Math. Monthly 98 (1991), 931-934. 


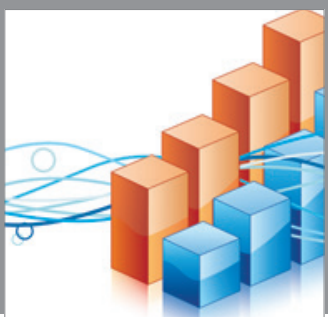

Advances in

Operations Research

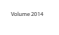

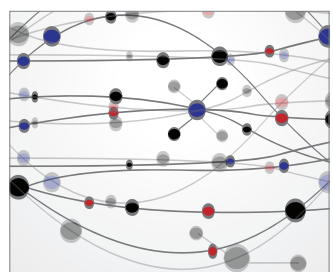

\section{The Scientific} World Journal
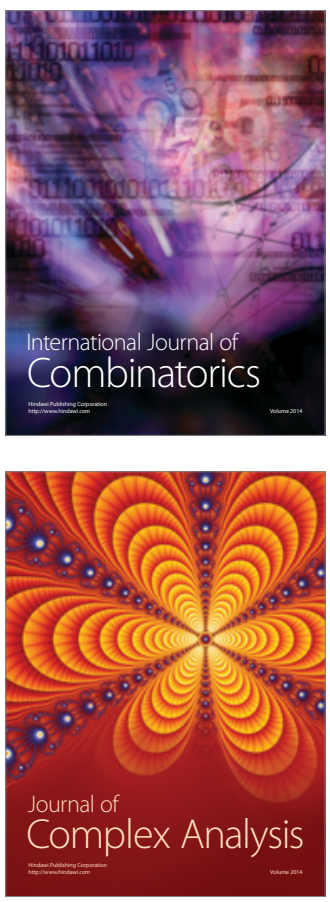

International Journal of

Mathematics and

Mathematical

Sciences
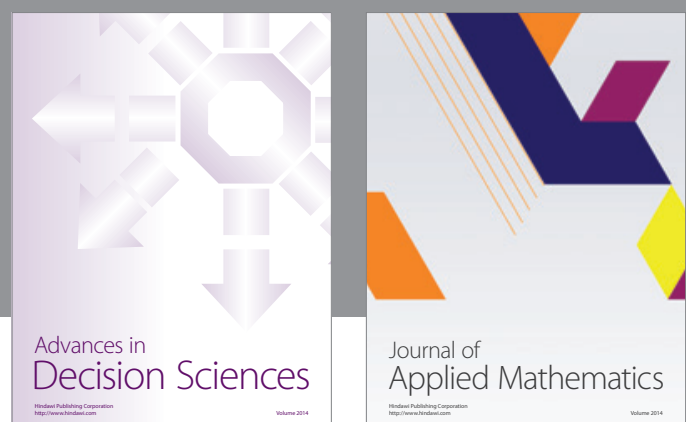

Journal of

Applied Mathematics
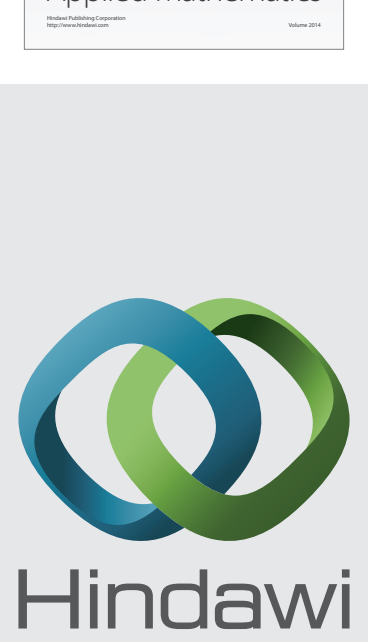

Submit your manuscripts at http://www.hindawi.com
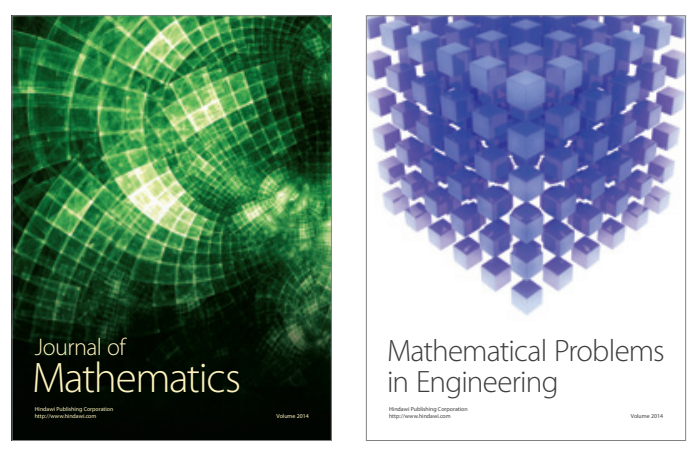

Mathematical Problems in Engineering
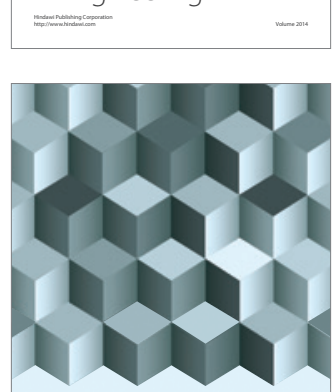

Journal of

Function Spaces
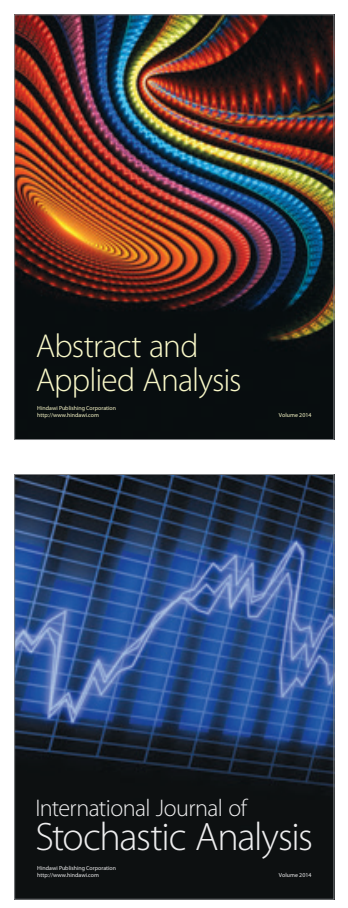

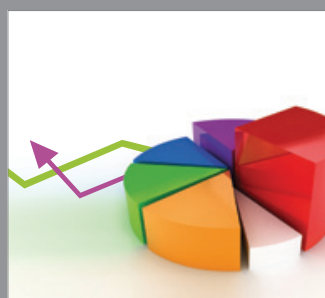

ournal of

Probability and Statistics

Promensencen
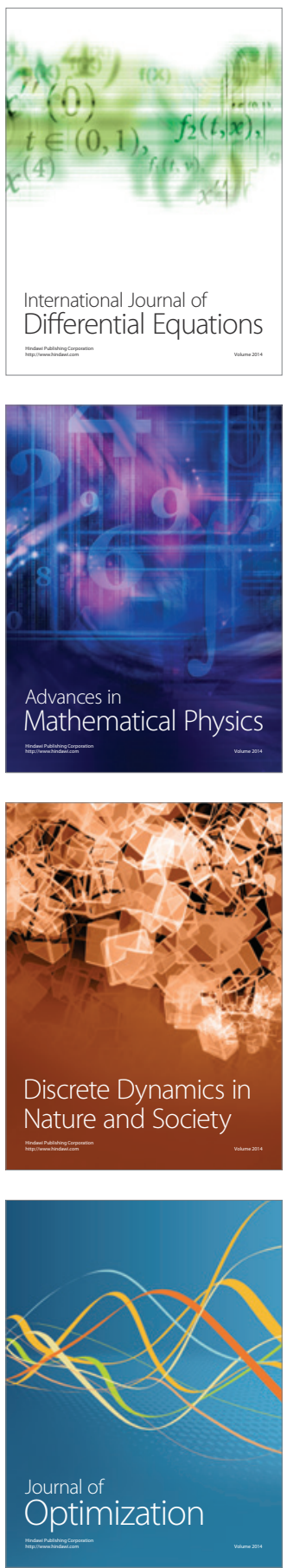